\title{
The Effect of Bicycle Ergometer Training on Serum Lipids Levels of Obese Middle School Students
}

\author{
JAEHA SEO ${ }^{1)}$, BYUNGJUN CHO ${ }^{2)}$ \\ 1) Department of Physical Education, Chungnam National University \\ 2) Department of Emergency Medical Technology, Kangwon National University: Kuydong Samchuock \\ City, Kangwondo, 245-711 South Korea.TEL: +82 33-570-6440,E-mail: cho6451@gmail.com
}

\begin{abstract}
Purpose] The purpose of this study was to examine the changes of the total cholesterol (TC), triglyceride (TG), high density lipoprotein cholesterol (HDL-C) and low density lipoprotein cholesterol (LDL-C) in the serum of obese middle school students after bicycle ergometer training. [Subjects and Methods] A total of 20 middle school students who had more than $21 \%$ body fat were recruited for this study. They were randomly assigned into one of two groups (exercise $=10$, control=10). The exercise group performed bicycle ergometer exercise three days per week for 40 minutes for eight weeks. Student's t-test was used to compare differences between the two groups. [Results] There was a significant decrease in TC in exercise group compared to the control. There was a significant decrease in TG in exercise group compared to the control. There was a significant increase in HDL-C and a significant decrease in LDC-C after the bicycle ergometer exercise program. [Conclusion] The results indicate that 8 weeks of bicycle exercise may improve the obesity of middle school students. Therefore, it is recommended that a physical training program is needed for youth to provide them with a healthy life.

Key words: Total cholesterol, Triglyceride, Exercise program
\end{abstract}

(This article was submitted May 9, 2011, and was accepted Jun. 29, 2011)

\section{INTRODUCTION}

If you disregard genetics, obesity is caused by an imbalance between calorie intake and consumption. Recent research has reported that physical inactivity is a major problem in obese children and adults who actually eat less than the required calorie intake per day ${ }^{1)}$. The relationship between exercise, blood lipid, and lipoprotein can be approached cross-sectional or longitudinal training studies. Cross-sectional studies mainly compare between active and inactive subjects. Generally speaking, active subjects have a higher level of high density lipoprotein cholesterol (HDL-C) and a lower level of low density lipoprotein cholesterol (LDL-C). This indicates a low risk of cardiovascular disease. On the other hand, longitudinal training studies are designed to investigate the effects of regular exercise blood lipid and lipoprotein. A previous study reported that aerobic exercise increased HDL-C and decreased LDL-C which was associated with a decrease in cardiovascular disease ${ }^{2)}$. Thus, regular exercise training may have a positive effect on blood lipid. Another study showed that running $24 \mathrm{~km}$ in a week significantly decreased triglyceride (TG) and total cholesterol levels (TC); however, the HDL-C level was significantly increased after exercise training ${ }^{3}$. Based on previous research, it has been concluded that athletes may have a higher HDL-C level than normal people because physical training causes increases TC, LDL-C, and TG ${ }^{4-6)}$. To the best of our knowledge, there have been many studies focusing on the changes of lipoprotein associated with adult diseases and the results of the present study further substantiate the findings of these other studies.

The purpose of the present study was to explore the effects of long-term exercise on the changes of blood lipid levels of obese middle school students to provide basic information for preventing adult diseases.

\section{SUBJECTS AND METHODS}

The participants for this study were middle school students who were attending C middle school in Daejeon, South Korea. A total of one hundred students were recruited and measured for a body composition. After the measurement, 20 students who had more than $21 \%$ fat were randomly assigned into two groups : an exercise group $(n=10)$ and control group $(n=10)$.

The exercise group performed eight weeks of exercise, and the control group did not participate in the regular exercise program. The subjects participated in this study with the consent of their parents, and written informed consent agreements were obtained from all subjects prior to the study. Table 1 shows the characteristics of the subjects. The exercise group performed regular exercises while the control group did not do any exercise except for regular physical education class. Before starting the exercise program, blood was taken for the analysis of TC, TG, HDL-C, and LDL-C. After the eight weeks of intervention, the same procedure was carried out. 
Table 1. Physical characteristics of subjects

\begin{tabular}{lcccc}
\hline Group & Age (yrs) & Height $(\mathrm{cm})$ & Weight $(\mathrm{kg})$ & Fat $(\%)$ \\
\hline Exercise $(\mathrm{n}=10)$ & $12.5 \pm 0.5$ & $164.6 \pm 7.8$ & $62.5 \pm 8.2$ & $23.2 \pm 1.0$ \\
Control $(\mathrm{n}=10)$ & $12.1 \pm 0.3$ & $162.7 \pm 8.4$ & $63.8 \pm 9.8$ & $23.7 \pm 0.8$ \\
\hline
\end{tabular}

Values are Means \pm Standard Deviations.

Table 2. Pre- and post-exercise triglyceride and cholesterol levels of the two group (Unit: $\mathrm{mg} / \mathrm{dl}$ )

\begin{tabular}{|c|c|c|c|c|c|}
\hline & \multicolumn{2}{|c|}{ Exercise Group } & \multicolumn{2}{|c|}{ Control Group } \\
\hline & & Pre & Post & Pre & Post \\
\hline \multirow{3}{*}{$\mathrm{TC}$} & M & $177.1^{*}$ & $170.8^{*}$ & 172.8 & 171.6 \\
\hline & SD & 10.59 & 8.00 & 5.83 & 6.70 \\
\hline & M & 113.8 & $98.1 *$ & 109.8 & 108.4 \\
\hline \multirow[t]{2}{*}{ TG } & SD & 10.49 & 32.05 & 10.06 & 6.47 \\
\hline & M & $46.1 * *$ & $52.7 * *$ & 46.5 & 47.9 \\
\hline \multirow[t]{2}{*}{ HDL-C } & SD & 2.77 & 3.27 & 3.21 & 5.20 \\
\hline & M & $145.09 * *$ & $138.64 *$ & 141.54 & 140.34 \\
\hline LDL-C & SD & 9.37 & 6.76 & 6.32 & 7.64 \\
\hline
\end{tabular}

On the data collection day, all the subjects arrived at the lab 20 minutes before the experiment and had a 20-minute rest. During this time their heart rate was measured. Then, the subjects performed a five-minute warm-up followed by a ten-minute rest. To measure the heart rate, a polar heart rate tester (GBR) was used. Astrand's graded exercise method was used because obese students had a low concentration and adaptation to the test. From the base line, the speed of the ergometer was increased every 2 minutes, and the total exercise time was 10 minutes. The bicycle ergometer training consisted of a five-minute warm up, 30 minutes of exercise, and a five-minute cool down. The endurance exercise training consisted of three days per week. The exercise session lasted 40 minutes for eight weeks. We checked the effects of aerobic exercise, exercise time and heart rate and recorded it for further analysis. The heart rate was measured for every minute, and \%HRmax was calculated according to Karvonen \& Vuorimaa (1988).

Because the blood lipid level is affected by the amount of food intake, all the subjects were not allowed to eat any food the day before the experiment. On the test day, $5 \mathrm{ml}$ of the blood was collected from the antecubital vein using a 10 $\mathrm{ml}$ needle by a nurse. The blood was collected in a heparinized tube, then centrifuged for 5 minutes. Before the analysis of the blood, it was kept in a freezer. The Green Cross Company performed the blood analysis. LDL-C was calculated using the following formula.

$\mathrm{LDL}-\mathrm{C}=\mathrm{TC}-(\mathrm{HDL}-\mathrm{C}+\mathrm{TG} / 5)$

Descriptive data are expressed as mean $\pm \mathrm{SD}$ for all variables. Student's t-test was used to find the significance of differences in all comparisons. Differences were considered significant at $\mathrm{p}<0.05$. Statistical analysis was conducted using SPSS for Windows ${ }^{\mathrm{TM}} 13.0$.

\section{RESULTS}

Table 2 presents the mean $\pm \mathrm{SD}$ values of TC, TG, HDL-C, and LDL-C. For TC, there was a significant decrease in the exercise group(pre-task $177.1 \pm 10.59$, posttask $170.8 \pm 8.00, \mathrm{p}<0.01$ ), but no difference was found in the control. For TG, no differences were found in the exercise group or the control group, but the exercise group showed a post-exercise in TG level which was significantly lower than that to the control $(\mathrm{p}<0.05)$.

For HDL-C, the exercise group showed a post-exercise increase of $14.32 \%$ pre-task $46.1 \pm 2.77$, post-task $52.7 \pm$ $3.27(p<0.05)$. No significant difference was found in the control. There was a significant difference between the two groups $(\mathrm{p}<0.05)$.

After the exercise program, the LDL-C level was reduced by $4.45 \%(p<0.05)$ in the exericise group, and there was a significant difference between the two groups $(\mathrm{p}<0.05)$.

\section{DISCUSSION}

This study investigated whether or not eight weeks of a bicycle ergometer exercise program would change TC, TG, HDL-C, and LDL-C in obese middle school students. Particularly, this study was designed to target obese students to prevent adult diseases. Furthermore, it provides basic information about exercise therapy. Cholesterol has an important role in the body as a precursor of growth hormones and 7-dehydrocholesterol, and it also helps to absorb lipids and vitamins. However, an excessive accumulation of cholesterol causes arteriosclerosis, hypertension, and cardiovascular diseases. An increased blood lipid level is a primary factor in coronary artery diseases. For adolescents, the normal range of TC is 120- 
$200 \mathrm{mg} / \mathrm{dl}$. It is well known that an aerobic exercise program can change the level of blood lipids. In this study, after the exercise program, the TC level was significantly reduced. This result agrees with those of other studies that have reported a decrease in $\mathrm{TC}^{4)}$. The reason for this is that the blood lipid level, exercise period, age, exercise intensity, body weight, and body composition would change the TC level. On the other hand, the control group did not show a significant decrease in the TC level, so concluded that the TC level was affected by the exercise training.

Regular physical activities will reduce the TG level of a cell in the skeletal and cardiac muscles. Aerobic exercises promote the oxidative process using fatty acids in the Krebs cycle. This also helps enzymes which are involved in the Krebs cycle and this enzyme activity leads the increased endurance time, creating an increased demand for oxygen ${ }^{7)}$.

Changes in TG level due to exercise differ with exercise intensity and amount, but it is well known that short-term exercise does not change the TG level. The results of the present study are consistent with those of a study ${ }^{8)}$ that reported changes in TG level following long -term exercise on three days per week. In the control group, the TG level also changed because the participants were very active and consumed a lot of calories during daily activities. Eating food and participating in a four week swimming program increased HDL-C levels ${ }^{9,10)}$. In the present study, the HDL-C increase was consistent with a 12 -week treadmill exercise $\mathrm{e}^{411-15)}$. For the control group, it can be demonstrated that the $3.01 \%$ increase in HDL-C was a time effect. LDL-C decreased dramatically in the exercise group, and a lower LDL-C level can reduce the risk of arteriosclerosis both in adolescents and adults.

\section{REFERENCES}

1) Wooley SC, Wooley OW, Dyrenforth SR: Obesity treatment reexamined: the case for a more tentative and experimental approach. NIDA Res Monogr, 1979, 25: 238-250.

2) Williams PT, Krauss RM, Wood PD, et al.: Lipoprotein subfractions of runners and sedentary men. Metabolism, 1986, 35: 45-52.

3) Wood PD: Plasma lipoprotein bistributios in male and female runners. Ann N V Acad Sci, 1977, 748-763.

4) Matsumoto I, Miyake Y, Mizukawa M, et al.: Impact of low-density lipoprotein cholesterol/high-density lipoprotein cholesterol ratio on longterm outcome in patients undergoing percutaneous coronary intervention. Circ J, 2011, 75: 905-910.

5) Bliar SN, Eoosworth NM, Flskell WI, et al.: Comparison of nurtriner intake in middle-aged men and women runners and controls. Med Sci Sports Exer, 1981, 13: 310-315.

6) Bostom AG: Lipid and Lipoprotein profiles relate to peak aerobic power in spinal cord injured men. Med Sci Sports Exerc, 1991, 23: 409-414.

7) Painelli VS, Roschel H, Gualano B, et al.: The effect of carbohydrate mouth rinse on maximal strength and strength endurance. Eur J Appl Physiol, 2011, 111: 2381-2386.

8) Cooper KH, Pollock ML: Physical fitness level vs selected coronary risk factors, a cross-sectional story. JAMA, 1976, 26: 166-169.

9) Berg A: Changes in HDL subfractions after a single extended episode of physical exercise. Atherosclerosis, 1983, 47: 231-240.

10) Brownell KD: Changes in plasma lipid \& lipoprotein levels in men and women alter a program of moderate exercise. Circulation, 1982, 65: 477484.

11) Adner MM, Casteeli WP: Elevated High - Density Lipoprotein levels in Marathon Runners. JAMA, 1980, 243: 534-536.

12) Goldberg LD, Elliot L, Schultz RW, et al.: Changes in lipid and lipoprotein levels after weight training. JAMA, 1984, 252: 504-506.

13) Quig DW: Effects of short-tern aerobic conditioning and high cholesterol feeding on plasma total and lipoprotein cholesterol levels in sedentary young man. Am J Clin Nutr, 1983, 38: 825-834.

14) Karvonen J, Vuorimaa T: Heart rate and exercise intensity during sports activities. Sports Med, 1988, 5: 303-311.

15) ACSM: ACSM Guidelines for exercise testing and prescription. 7th edition. Philadelphia: Lippincott Williams and Wilkins, 2006, pp 10-150. 